\title{
Le transport hippomobile urbain au Sénégal - Situation et importance économique dans la ville de Thiès
}

\author{
C. Ly ${ }^{1 *}$ B. Fall ${ }^{2}$ B. Camara ${ }^{3}$ C.M. Ndiaye ${ }^{4}$
}

\section{Mots-clés}

Cheval - Transport - Zone urbaine Valeur économique - Véhicule à traction animale - Charrette - Attelage Analyse coût avantage - Sénégal.

\section{Résumé}

Cette étude décrit la situation et analyse l'importance économique de la traction hippomobile urbaine et périurbaine au Sénégal, à partir du cas de la ville de Thiès. Cent attelages (64 fiacres et 36 charrettes) ont été choisis au hasard et leurs cochers ont été interrogés. Les données collectées ont concerné les cochers et les chevaux, les caractéristiques et la gestion des attelages et enfin les résultats économiques (coûts, recettes et gains). Pour un travail de six jours par semaine, les fiacres et les charrettes ont généré pour le propriétaire de l'attelage un gain monétaire net quotidien moyen équivalent respectivement à 61 p. 100 (2 202 francs CFA) et 66 p. 100 (2 779 FCFA) de leur chiffre d'affaires quotidien respectif (3 600 et 4200 FCFA). Les charrettes sont apparues légèrement plus rentables car leurs tarifs étaient plus élevés. L'importance économique du cheval est illustrée et des recommandations sont proposées pour améliorer ses performances et sa participation à l'économie nationale.

\section{INTRODUCTION}

Au Sénégal, le cheval est présent dans plusieurs secteurs d'activités telles que la traction hippomobile urbaine et rurale, l'industrie des courses hippiques, l'équitation sportive ou d'agrément et, dans une moindre mesure, la chorégraphie équine et la boucherie hippophagique. Au niveau ethnologique, les chevaux sénégalais ont une origine qui a déjà été documentée $(2,5,6,7,13,14,15,16)$.

Les effectifs de chevaux du Sénégal ont été estimés officiellement à près de 434000 têtes en 1993 dont près de 80 p. 100 dans le bassin arachidier (3). Fielding (11) estime les mêmes effectifs à 1,2 p. 100 du cheptel chevalin africain, soit seulement 208000 têtes avec une tendance à la baisse entre 1980 et 1988. Après l'Ethiopie, le Sénégal est le cinquième pays africain en termes d'effectifs chevalins (9).

La majorité du cheptel équin sénégalais se situe dans le Nord et le Centre-Ouest du pays. Au Sud, la trypanosomose animale africaine constitue une contrainte sanitaire majeure bien que les

1. Service d'économie rurale et gestion, Ecole inter-états des sciences et médecine vétérinaires (EISMV), BP 5077, Dakar, Sénégal

2. Bureau du cheval, Direction de l'élevage, Dakar, Sénégal

3. Inspection régionale de l'élevage, Thiès, Sénégal

4. Ecole nationale des cadres ruraux (ENCR), Bambey, Sénégal

* Auteur pour la correspondance

Tél. : +2218256692; fax : +2218254383

E-mail : chly@syfed.refer.sn infiltrations de chevaux deviennent progressivement plus nombreuses et durables car elles sont favorisées par l'assèchement du climat et la progression des zones cultivées. La peste équine est endémique dans le Centre-Ouest du pays et on note aussi la présence du tétanos, de la lymphangite épizootique et du botulisme équin $(1,3,8,15)$.

Avec une dispersion limitée, les poneys du Sénégal sont différenciés en deux types, le Mpar et le Mbayar respectivement cheval du Cayor et cheval du Baol, en référence aux deux régions centrales du Sénégal dont ils sont autochtones $(5,6)$. Epstein (7) signale que le moment de l'arrivée des poneys au nord de l'Afrique équatoriale est incertain et il serait plutôt favorable à l'idée d'introductions de différentes origines à différentes époques. En se basant sur les flux migratoires anciens, il accrédite aussi la thèse selon laquelle les poneys de l'Afrique de l'Ouest sont des descendants dégénérés d'anciens chevaux égyptiens de type Aryen ou Arabe et introduits lors des migrations Peules. Un environnement difficile dans les terres inondées du Niger ainsi que dans les zones subguinéennes et côtières ouest-africaines suscita une évolution qui aurait contribué à la réduction de la taille des produits. De plus, sur le substrat génétique très ancien qualifié d'oriental se seraient surajoutés des chevaux Barbes à partir de l'Afrique du Nord et des chevaux Dongolaw provenant de l'Est (7).

Selon Doutressoulle (5), les caractères du cheval Mpar ou du Cayor ont presque disparu au profit du Mbayar qui est plus grand. Ainsi, les chevaux autochtones utilisés pour le trait au Sénégal sont issus d'un fort métissage Mbayar X Mpar. Les chevaux de trait les plus courants sont des chevaux de petit format avec une 
hauteur au garrot entre 1,25 et $1,40 \mathrm{~m}$ et un poids de $200-250 \mathrm{~kg}$ (figure 1) (13). Ils sont très endurants, sobres et rustiques. Ndiaye (15) a estimé leur puissance moyenne à 420 watts en tenant compte d'une compacité moins élevée que pour les chevaux de trait européens.

Le cheval du Sahel qui descend du cheval Barbe a donné une variante dite cheval Fleuve, appelé Narou goor. On le retrouve parmi les chevaux de course et d'équitation. La présence du cheval Foutanké, du nom du Fouta ou vallée du Fleuve Sénégal, est également décrite comme le résultat d'un croisement entre le cheval du Sahel qui dérive du type mongolique Barbe et la jument poney de type Mbayar (6, 7). Il faut enfin noter l'existence de quelques chevaux de sang dans les cercles hippiques dakarois et à l'Escadron monté de la Gendarmerie nationale du Sénégal.

Avec l'introduction des voitures hippomobiles par les colons français, une autre forme d'utilisation du cheval s'est développée au Sénégal. Suite à des tests de faisabilité et de vulgarisation entre 1955 et 1965, les tractions équine et asine se sont maintenues malgré la promotion de la culture attelée au moyen de la traction bovine qui avait l'appui du programme Agricole (matériel et paires de bœufs à crédit). La rapidité du cheval et la diffusion de la charrette équine ont facilité l'exécution des opérations culturales, du transport et de l'exhaure (10). Ainsi, le cheval de trait agricole et la traction hippomobile jouent toujours un rôle fondamental dans les relations et les échanges ruraux, la commercialisation des produits agricoles, l'approvisionnement en intrants et produits de consommation et le transport de personnes.

Dans les pays en développement, le cheval, en plus de ses fonctions traditionnelles, constitue une alternative accessible comme moteur de fiacres-taxis ou de charrettes pour le transport de fardeaux, de matériaux de construction, d'ordures domestiques, etc. La forte présence de la traction hippomobile à Dakar et dans toutes les villes secondaires du Sénégal doit permettre au cheval et à la traction hippomobile d'être repris parmi les thèmes de la recherche-développement pour une meilleure efficacité dans l'exploitation des ressources animales. En 1987, un Bureau du cheval a été créé pour coordonner les interventions de l'Etat dans la promotion du cheval, le soutien à la traction hippomobile et la participation à la gestion des courses hippiques.

L'objectif général de ce travail a été de décrire et d'analyser la traction hippomobile au Sénégal, à partir du cas de Thiès, ville du Sénégal où les charrettes et les fiacres jouent un rôle économique indéniable, afin de renforcer les options basées sur l'utilisation du cheval et de contribuer aux efforts déployés par le Bureau du cheval de la Direction de l'élevage du Sénégal pour organiser et promouvoir, en l'occurrence, une meilleure exploitation du cheval de trait.

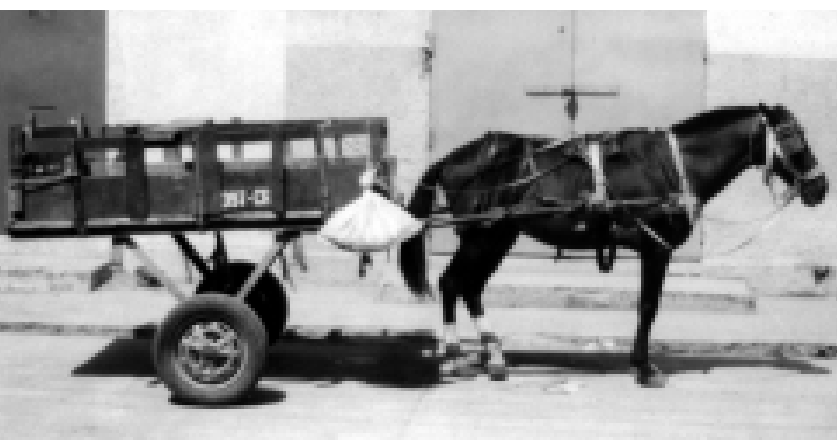

Figure 1 : charrette.
La ville de Thiès, à 70 km à l'est de Dakar, est une importante agglomération (170 000 habitants) où populations rurale et urbaine ont des relations d'échanges permanentes et très étroites. Située au nord du bassin arachidier sénégalais, Thiès est aussi le second pôle industriel du Sénégal et la demande en transport urbain est importante compte tenu de l'exode rural et de la stagnation des infrastructures de transport urbain. La demande en transport hippomobile est plus variée qu'à Dakar où le transport concerne plus les matériaux de construction, les ordures ménagères et les effets domestiques que les personnes.

\section{MATERIEL ET METHODES}

Cent attelages hippomobiles ont été choisis à Thiès, au hasard, pendant les mois de juillet et d'août 1995. Leurs conducteurs ou propriétaires ont été soumis à un questionnaire d'enquête rétrospectif lors d'entretiens menés pendant la pause au point de stationnement ou de repos. La méthodologie suivie pour cette étude a été affinée à partir d'une première enquête sur le même thème à Rufisque (40 km de Dakar) en 1994 (1). Cette première enquête avait permis d'identifier les contraintes du transport hippomobile urbain et de déterminer les postes de dépenses et les types de recettes. Les points suivants soumis à discussion ont concerné les aspects techniques et socio-économiques du transport hippomobile :

- profil des charretiers et propriétaires ;

- caractéristiques des animaux (âge, sexe, race, état général) ;

- élevage (rations alimentaires, hygiène de travail, maréchalerie et matériel de pansage);

- pathologies (maladies et affections courantes, moyens de couverture sanitaire et de prophylaxie, automédication);

- attelages (types, état, harnachement, coûts d'investissement, d'entretien et de renouvellement, pneumatiques, durée de vie du matériel, charges transportées, types de chargement, moyens de signalisation, catadioptres, etc.) ;

- gestion (organisation du transport, frais encourus, structure des prix pour les services rendus, relations avec les structures publiques communales, policières et vétérinaires).

Des enquêtes complémentaires ont été également menées au niveau du marché rural hebdomadaire de Touba-Toul, à $40 \mathrm{~km}$ de Thiès, afin de recueillir des informations sur la filière de commercialisation des chevaux (organisation et prix) auprès de vendeurs de chevaux. Des entretiens ont été parallèlement conduits dans les écuries et avec les professionnels de la filière hippomobile : fabricants de voitures hippomobiles et de harnais, maréchaux-ferrants et vendeurs d'aliments pour chevaux.

Les données ont été analysées grâce au logiciel SPSS (18) après constitution d'une base de données. L'accent a été mis sur les statistiques descriptives.

\section{RESU LTATS}

\section{Identification des cochers}

A Thiès, les calèches prédominaient très largement. Les enquêtes ont concerné 64 calèches et 36 charrettes soit 14 p. 100 des 700 attelages estimés. Les proportions étaient proches de celles de Camara (1) pour la ville de Rufisque : 87 calèches et 24 charrettes, ce qui représentait 26 p. 100 des attelages immatriculés. 
La répartition des ethnies des conducteurs était proche de celle du bassin arachidier avec 60 p. 100 de Wolofs, 21 p. 100 de Sérères et 13 p. 100 de Peuls. La ville de Thiès et ses environs étaient les lieux d'origine de 72 p. 100 des cochers. Dans 65 p. 100 des cas, les cochers étaient des chefs de famille. Cinquante-deux pour cent avaient entre 21 et 40 ans (moyenne : $31 \pm 6$ ans), 28 p. 100 avaient plus de 40 ans (moyenne : $50 \pm 6$ ans) et 20 p. 100 étaient des mineurs. Les cochers interrogés étaient soit des cochers propriétaires soit de simples cochers embauchés (tableau I). Les propriétaires étaient des résidents permanents à Thiès alors que les cochers étaient plutôt des saisonniers $(\mathrm{p} \leq 0,05)$. Les propriétaires non cochers étaient des fonctionnaires ou venaient du secteur privé et avaient d'autres activités (vendeurs d'aliments pour bétail et chevaux, commerçants, divers).

\section{Les chevaux}

L'âge moyen des chevaux était de $8 \pm 2$ ans, avec un minimum de 3 ans et un maximum de 17 ans $(n=100)$. Les chevaux étaient en service depuis $3 \pm 3$ ans avec un minimum d'un an et un maximum de 10 ans. Une proportion de 29 p. 100 des chevaux était en service depuis plus de 5 ans (tableau II). Ni jument ni hongre n'ont été recensés dans l'échantillon.

Le commerce des chevaux se faisait dans les marchés hebdomadaires ruraux. Les statistiques officielles sur les exportations ont fourni peu d'informations sur les échanges et les flux frontaliers

\section{Tableau I}

Statut et type de résidence des cochers

\begin{tabular}{lccc} 
& \multicolumn{3}{c}{ Type de résidence } \\
\cline { 2 - 4 } Statut & Permanente & Temporaire & Total \\
\hline Conducteurs & 24 & 7 & 31 \\
Propriétaires conducteurs & 64 & 5 & 69 \\
Total & 88 & 12 & 100
\end{tabular}

$\chi^{2}=4,76 ; \mathrm{p} \leq 0,05$

\section{Tableau II}

Durée d'utilisation des chevaux

\begin{tabular}{lccccc} 
D urée en années & $\mathbf{1}$ & $\mathbf{2}$ & $\mathbf{3}$ ou 4* & $\mathbf{5}$ & $>\mathbf{6}$ \\
\hline \% de chevaux & 37 & 18 & 16 & 17 & 12 \\
* regroupées dans le questionnaire & & &
\end{tabular}

Tableau III

Catégories de prix d'achat des chevaux et âge $(n=85)$

$\begin{array}{lccr}\begin{array}{l}\text { Catégories de prix } \\ \text { (FCFA*) }\end{array} & \begin{array}{c}\text { Prix moyen } \pm \text { ET** } \\ \text { (FCFA*) }\end{array} & \begin{array}{c}\text { Age moyen } \\ \pm \text { ET** }\end{array} & \begin{array}{c}\text { \% des chevaux } \\ 25000-50000\end{array} \quad 43194 \pm 6777 \\ 55000-75000 & 64233 \pm 7477 & 7,6 \pm 2,3 & 42,3 \\ 80000-100000 & 90429 \pm 9741 & 7,9 \pm 2,6 & 35,3 \\ 110000-150000 & 134000 \pm 11937 & 7,5 \pm 2,5 & 16,5 \\ \end{array}$

$*$ FCFA $=$ francs CFA

** ET = écart-type étaient très limités. Les lieux d'achat qui ont été signalés étaient les deux grands marchés traditionnels de Touba-Toul et de DahraDjolof respectivement à 30 et $200 \mathrm{~km}$ de Thiès. Le prix d'achat moyen était de $63741 \pm 25563$ FCFA $(n=85)$. Les catégories de prix sont présentées dans le tableau III. La dispersion des prix était importante. C'était le format du cheval et son état général qui ont conditionné son prix. Les chevaux les plus chers avaient une moyenne d'âge inférieure aux catégories de chevaux meilleur marché avec 5,8 ans contre 7,5 à 7,9 ans en moyenne (tableau III). La location a concerné 16 p. 100 des chevaux qui étaient loués au tarif journalier de $982 \pm 508$ FCFA $(n=14)$, la nourriture étant à la charge du propriétaire. Les chevaux étaient achetés dans 81 p. 100 des cas. La plupart des chevaux recensés provenaient des environs de Thiès (37 p. 100).

Le temps de travail par jour était de $7 \pm 2 \mathrm{~h}(\mathrm{n}=100)$ et 59 p. 100 des cochers ont déclaré avoir un jour régulier de repos qui était généralement le dimanche. Il pouvait y avoir deux chevaux pour un attelage au niveau des lieux de parcage afin de remplacer les chevaux malades ou d'augmenter le nombre de courses en cas de forte demande (veilles et jours de fêtes, fins de mois).

\section{La conduite des chevaux}

Les chevaux étaient parqués en plein air sur des terrains vagues non clôturés. Des bassines et des fûts découpés faisaient office de mangeoires et d'abreuvoirs. Deux à trois repas étaient donnés par jour avant et après le travail ou à la pose de la mi-journée. Les rations alimentaires étaient très hétérogènes et leur composition variait selon les saisons et les produits disponibles (fane d'arachide, foin, son, mil, niébé, maïs et herbe en hivernage). Certains chevaux étaient complémentés avec un aliment industriel à base de remoulage ou ripasse qui était vendu par sac de $40 \mathrm{~kg}$ à 3000 FCFA. Le coût journalier de la ration a été estimé à $1473 \pm 435$ FCFA en saison sèche et $1184 \pm 527$ FCFA en hivernage pendant lequel l'herbe verte était disponible. Les valeurs modales étaient de 600 FCFA par jour en saison des pluies et de 1130 FCFA par jour en saison sèche.

L'alimentation était relativement chère car le cours de la fane d'arachide, l'une de ses principales composantes, était élevé en zone urbaine, à raison de 1200 FCFA le sac de $15 \mathrm{~kg}$, soit 80 FCFA $/ \mathrm{kg}$. La fane d'arachide était en effet devenue une spéculation très lucrative du fait de sa forte demande en zone urbaine, conséquence de l'importance prise par l'élevage citadin ovin. Par ailleurs, le mil et surtout le maïs avaient également des cours élevés, avec respectivement 140-175 et 150-200 FCFA/kg. Si une ration journalière de $4 \mathrm{~kg}$ de mil et de $4 \mathrm{~kg}$ de fane d'arachide couvrait passablement les besoins pour le travail et l'entretien du cheval, son coût moyen s'élevait déjà à 950 FCFA. 
Au moment de l'enquête, tous les chevaux de l'échantillon étaient en activité normale et il n'a pas été fait d'examen clinique pour caractériser de manière précise leur état sanitaire. Les maladies les plus fréquentes ont été recensées à partir des dires des cochers (tableau IV). La gourme et les myosites ont constitué la grande majorité des cas rapportés, soit 68 p. 100. Le tableau pathologique peut être complété par les plaies cutanées et les affections oculaires (4).

Les soins étaient pris en charge par les propriétaires de chevaux et les traitements traditionnels les plus divers ont été rapportés : bière et limonades gazeuses pour les coliques; inhalations de fumée de tabac ou de bouse de vache séchée pour la gourme; feuilles de manguier ou de papayer pour le tétanos. Les myosites étaient traitées par application locale de vinaigre ou de beurre de karité (Butyrospermum paradoxum subst. parkii).

Parmi les cochers interrogés, 87 p. 100 ont déclaré être en contact avec le Service régional de l'élevage ou savaient que les visites étaient indispensables. Pour les vaccinations, 82 p. 100 des cochers ont déclaré que leur cheval était vacciné contre la peste équine et 60 p. 100 contre le tétanos. Les coûts de la vaccination étaient respectivement pour la peste équine et le tétanos 1000 et 1500 FCFA.

Malgré les frais encourus par les propriétaires, la conduite des chevaux s'est caractérisée par des conditions d'hygiène et d'alimentation généralement précaires. Les lieux de repos étaient le plus souvent des endroits insalubres. Les terrains communaux étaient occupés illégalement. Ils pouvaient aussi être «prêtés » aux propriétaires, sans frais particulier.

\section{Le matériel de pansage et la maréchalerie}

Le matériel de pansage, composé de brosses, de chiffons, d'éponges et de morceaux de filets de pêche, était donc rudimentaire. Les fibres de mbarbëf (Momordica charantia) ont souvent été utilisées pour enlever les croûtes. Le pansage était régulier et a coïncidé avec les manipulations occasionnées par le bain quotidien avec une bassine d'eau de 251 qui coûtait 50 FCFA.

Tous les chevaux portaient des fers et 92 p. 100 des fers observés étaient dans un état bon ou acceptable. Le renouvellement s'effectuait tous les six jours à un mois soit, en moyenne, $13 \pm 7$ jours avec un coût unitaire de 200 ou 250 FCFA, suivant le type de pointes utilisées (moyenne : $230 \pm 65$ FCFA ; $n=97$ ).

\section{Les attelages}

Au Sénégal, le terme «charrettes » désigne à la fois les calèches (fiacres ou encore « voitures ») et les charrettes proprement dites. Les charrettes sont des plates-formes à ridelles aménagées pour le transport de fardeaux et accessoirement de personnes (figure 1). Les fiacres sont aménagés pour le transport de personnes et accessoirement de fardeaux (figure 2). Les attelages sont toujours individuels.

Au moment de leur acquisition, le coût maximum des attelages était de 110000 FCFA, avec pour les calèches une moyenne de $50595 \pm 18128$ FCFA $(n=47)$ et pour les charrettes une moyenne de $47286 \pm 20652$ FCFA $(n=35)$. Ces coûts moyens ont concerné des attelages d'occasion, réparés par les ferronniers, car les attelages neufs étaient pratiquement absents du marché. La durée normale d'utilisation des attelages a été estimée par leurs propriétaires à six ans mais le parc des attelages était particulièrement vétuste, surtout en ce qui concerne les calèches, et la fréquence des réparations était très élevée. Bien que l'entretien régulier ait été évalué pour l'année à $2119 \pm 1082$ FCFA $(\mathrm{n}=21), 79$ p. 100 des cochers ont reconnu ne pas y veiller.
Tableau IV

Maladies rapportées par les cochers

\begin{tabular}{lcc}
$\begin{array}{l}\text { Maladies citées } \\
\text { par les cochers }\end{array}$ & $\begin{array}{c}\text { N b de citations } \\
\text { par les cochers }\end{array}$ & $\begin{array}{c}\text { \% de citations } \\
\text { par les cochers }\end{array}$ \\
\hline Gourme & 39 & 34,8 \\
M yosites/fourbures & 37 & 33,0 \\
Coliques & 22 & 19,6 \\
Lymphangite & 12 & 10,8 \\
Tétanos & 2 & 1,8 \\
Total & 112 & 100
\end{tabular}

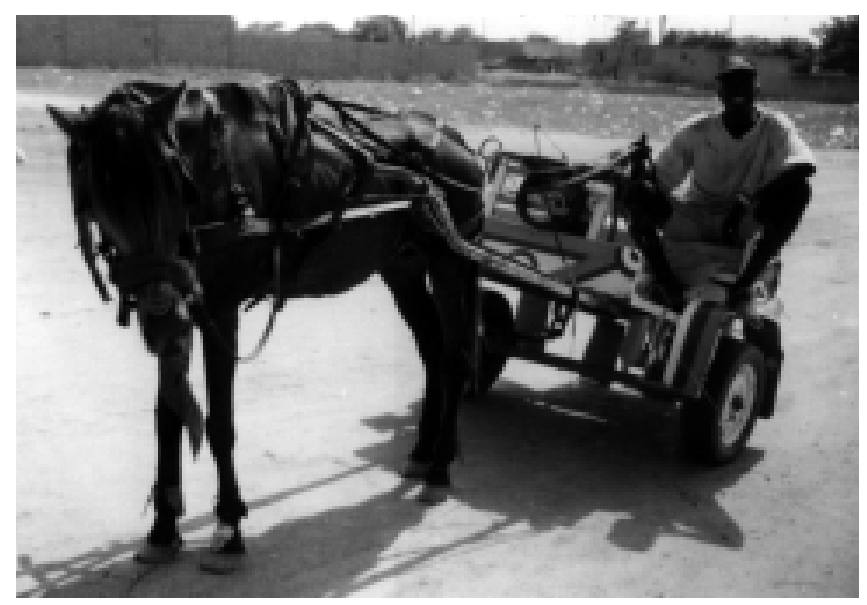

Figure 2 : calèche.

\section{Le harnachement et les accessoires}

Le harnachement était une bricole (11). Les brancards étaient supportés par des sangles de soutien solidaires d'une sellette placée en arrière du garrot (dossière) et le tout était fixé au thorax de l'animal par une sangle (sous-ventrière). Dans certains cas, le harnachement était complété par une croupière et un reculement ou fessière (avaloire).

Les harnais étaient fabriqués avec une forte proportion de matériaux synthétiques de récupération (pneus, chambres à air, sacs de riz, plastiques). Le cuir était très peu utilisé et seulement 7 p. 100 des harnais étaient en cuir. Sur la base de leur aspect extérieur et du rapieçage, 68 p. 100 des harnais ont été jugés bons ou acceptables contre 32 p. 100 qui étaient en très mauvais état. Le coût moyen d'un harnais était de $7193 \pm 2608$ FCFA $(n=95)$. La durée de vie moyenne a été estimée par les conducteurs à $15 \pm 9$ mois $(\mathrm{n}=99)$.

Les accessoires des attelages étaient aussi rudimentaires et généralement d'occasion. Les pneus toujours usés étaient obtenus à 2000 FCFA pièce pour une durée d'utilisation moyenne de $7 \pm 6$ mois $(\mathrm{n}=85)$. La signalisation était inexistante hormis l'avertisseur acquis à $1430 \pm 499$ FCFA $(n=23)$. La cravache était artisanale et coûtait $218 \pm 189$ FCFA $(n=96)$.

Il faut souligner que 82 p. 100 des cochers ont déclaré ne pas circuler la nuit, où le système d'éclairage le plus courant était celui de la lampe tempête à pétrole. Les torches à piles étaient rarement utilisées et l'équipement d'éclairage revenait en moyenne à 1400 \pm 558 FCFA $(n=15)$. 


\section{Les recettes}

Le nombre quotidien de courses était de $12 \pm 3$ et de $7 \pm 5$, respectivement pour les fiacres et pour les charrettes. Pour les courses en fiacre, le tarif moyen d'une course était de $210 \pm 158$ FCFA avec un minimum à 100 FCFA et un maximum à 800 FCFA. Pour les charrettes, le tarif moyen d'une course était de $393 \pm 202$ FCFA avec un minimum à 150 FCFA et un maximum à 1000 FCFA. A titre de comparaison, les taxis urbains faisaient payer la course 375 FCFA, quelle que soit la distance. Les taxis dits « clandos » qui faisaient des trajets sans détour vers le grand marché de Thiès à partir d'une station de départ dans chaque quartier avaient un tarif fixé à 75 FCFA par personne alors que le transport en commun par « car rapide » revenait à 50 FCFA par personne.

Les tarifs par course étaient relativement stables quand on examine les catégories de recettes déclarées par les cochers après les inévitables négociations et marchandages (tableau V). Les cochers non propriétaires versaient une somme quotidienne au propriétaire du cheval et de l'attelage qui prenait en charge la nourriture $\mathrm{du}$ cheval. Le versement moyen calculé était de l'ordre de $1396 \pm 652$ FCFA $(n=45)$ mais la valeur modale était de 1500 FCFA par jour.

\section{Les relations avec les structures officielles}

La réglementation du transport hippomobile a été fixée officiellement suivant un arrêté du conseil municipal après avis des responsables techniques et des propriétaires de chevaux. Chaque cheval devait faire l'objet de visites sanitaires et un carnet signalétique sanitaire devait être tenu et présenté à la demande des services de l'élevage et de la sécurité routière. Les contrôles de police étaient peu appréciés par les cochers et les contraventions de 800 à 3000 FCFA pour circulation sur route nationale, non respect du code de la route, surcharges, etc. étaient souvent jugées arbitraires et élevées. Parmi les cochers interrogés, 98 p. 100 ont déclaré s'acquitter de la taxe municipale obligatoire de 100 FCFA par jour pour les charrettes et 2000 FCFA par mois pour les calèches. Cette différence dans le mode de taxation était un choix de la municipalité de Thiès qui enregistrait et contrôlait plus facilement les calèches au niveau du bureau de perception que les charrettes qui étaient jugées moins fixes et ainsi plus difficiles à contrôler. Elles étaient de ce fait imposées chaque jour par les collecteurs de taxe comme pour le petit commerce et les étals de marché.

Alors que 450 fiacres étaient immatriculés chaque année, il a été difficile d'évaluer le nombre exact de charrettes en circulation à Thiès car elles n'étaient pas immatriculées. De plus, les difficultés d'accès aux relevés des versements effectués au titre de la taxe sur les charrettes n'ont pas permis de procéder à des estimations. Cependant, en respectant les proportions rencontrées lors de l'en- quête, il peut être estimé que pour 100 attelages, il y avait environ 36 charrettes, d'où une estimation de 250 charrettes, soit un total estimé à 700 attelages à Thiès.

\section{Estimation des gains et de la rémunération du travail}

Un compte d'exploitation type permet de montrer que le transport hippomobile était une activité à forte intensité de main d'œuvre rémunérée. Les entrées et les sorties monétaires ont été estimées à partir des résultats moyens présentés et rapportés à un exercice d'un an. Les valeurs modales ont été utilisées quand elles apparaissaient plus fiables. Des amortissements linéaires ont été calculés pour les investissements et tous les comptes ont été faits pour un cheval par attelage (tableau VI). Lorsque le prix d'achat du cheval qui a une forte dispersion est relativement élevé, il a un impact important sur l'amortissement de l'attelage. Les chevaux dont le prix d'achat était supérieur à 110000 FCFA n'ont constitué que 6 p. 100 des chevaux dont l'âge et le prix d'achat ont été relevés.

\section{DISCUSSION}

\section{Gestion des chevaux et des attelages}

La forte proportion des propriétaires parmi les cochers interrogés pouvait être liée au fait que l'enquête s'est déroulée en début d'hivernage quand beaucoup de jeunes ruraux, parmi lesquels se recrutaient les cochers temporaires, étaient retournés aux champs. De plus, il n'y avait pas de chevaux provenant directement des exploitations agricoles. Cette situation s'explique par la spécialisation des chevaux affectés à la traction urbaine bien que leur format ait réduit les performances de traction et limité le poids des chargements.

L'entretien des chevaux a laissé à désirer et il a contribué à augmenter les risques d'indisponibilité, aggravés par l'exposition à la pluie et à la poussière dans les points de repos, le coût de l'alimentation et le défaut de rationnement en fonction des efforts fournis. Une meilleure alimentation ou de meilleurs soins, plus coûteux, n'auraient cependant pas apporté de recette supplémentaire, ni de gain sensible sur la longévité des animaux. En revanche, les fers étaient couramment utilisés à cause de la fréquence des trajets sur les principaux axes routiers de Thiès qui étaient tous bitumés.

L'état des harnais était souvent à l'origine de plaies diverses par compressions et frottements et il pouvait limiter la bonne exploitation de la force de traction des chevaux et leur maniabilité dans la circulation urbaine. La pharmacopée vétérinaire traditionnelle était utilisée bien que certains traitements empiriques aient été discutables surtout ceux contre la gourme et les coliques. Le mauvais état général des attelages était lié aux routes défectueuses, à leur

\section{Tableau V}

Tarifs des courses (FCFA)

$\begin{array}{llcc}\text { Catégorie de recette/course } & \text { Attelage } & \text { Moyenne } \pm \text { ET* } & \mathbf{n} \\ \text { Recette minimum } & \text { Fiacre } & 249 \pm 93 & 63 \\ & \text { Charrette } & 369 \pm 143 & 36 \\ \text { Recette moyenne } & \text { Fiacre } & 321 \pm 122 & 62 \\ & \text { Charrette } & 515 \pm 250 & 36 \\ \text { Recette maximum } & \text { Fiacre } & 497 \pm 221 & 63 \\ & \text { Charrette } & 928 \pm 468 & 36\end{array}$

* ET = écart-type 
Tableau VI

Compte d'exploitation type pour un fiacre et une charrette à Thiès

\begin{tabular}{|c|c|c|c|c|}
\hline \multirow[b]{2}{*}{ Rubriques } & \multicolumn{2}{|c|}{ Fiacre } & \multicolumn{2}{|c|}{ Charrette } \\
\hline & $\begin{array}{l}\text { Montant } \\
\text { FCFA* }\end{array}$ & $\begin{array}{c}\text { Pourcentage } \\
\text { du chiffre d'affaires }\end{array}$ & $\begin{array}{l}\text { Montant } \\
\text { FCFA* }\end{array}$ & $\begin{array}{l}\text { Pourcentage } \\
\text { du chiffre d'affaires }\end{array}$ \\
\hline 1. Alimentation/abreuvement/bain & & & & \\
\hline $\begin{array}{l}7 \text { mois de rations à } 1130 \text { FCFA/j (212 jours) } \\
5 \text { mois de rations à } 600 \text { FCFA/j (153 jours) } \\
2 \text { bassines d'eau à } 50 \text { FCFA/jour } \\
\text { Sous-total } 1\end{array}$ & $\begin{array}{r}239560 \\
91800 \\
36500 \\
367860\end{array}$ & $\begin{array}{r}21,3 \\
8,2 \\
3,2 \\
32,8\end{array}$ & $\begin{array}{r}239560 \\
91800 \\
36500 \\
367860\end{array}$ & $\begin{array}{r}18,3 \\
7,0 \\
2,8 \\
28,1\end{array}$ \\
\hline $\begin{array}{l}\text { 2. Frais annuels } \\
\text { Taxe communale } \\
\text { Pneus ( } 2 \text { paires à } 4000 \text { FCFA) } \\
\text { Fers ( } 4 \text { fers à } 800 \text { FCFA tous les } 15 \text { jours) } \\
\text { Entretien trimestriel attelage } \\
\text { Frais vétérinaires }\end{array}$ & $\begin{array}{r}24000 \\
8000 \\
20800 \\
8476\end{array}$ & $\begin{array}{l}2,1 \\
0,7 \\
1,9 \\
0,8\end{array}$ & $\begin{array}{r}31200 \\
8000 \\
20800 \\
8476\end{array}$ & $\begin{array}{l}2,4 \\
0,6 \\
1,6 \\
0,6\end{array}$ \\
\hline $\begin{array}{l}\text { - vaccination contre la peste } \\
\text { - vaccination contre le tétanos } \\
\text { - autre soins (provisions) } \\
\text { Cravache (1 par trimestre) } \\
\quad \text { Sous-total } 2\end{array}$ & $\begin{array}{r}1000 \\
1500 \\
1000 \\
3488 \\
68264\end{array}$ & $\begin{array}{l}0,1 \\
0,1 \\
0,1 \\
0,3 \\
6,1\end{array}$ & $\begin{array}{r}1000 \\
1500 \\
1000 \\
3488 \\
75464\end{array}$ & $\begin{array}{l}0,1 \\
0,1 \\
0,1 \\
0,3 \\
5,8\end{array}$ \\
\hline Total des charges monétaires & 436124 & 38,8 & 443324 & 33,8 \\
\hline $\begin{array}{l}\text { 3. Amortissement } \\
\text { Cheval (sur } 6 \text { ans) } \\
\text { Charrette ou fiacre (sur } 6 \text { ans) } \\
\text { H arnais (sur } 2 \text { ans) } \\
\text { Klaxon (sur } 2 \text { ans) } \\
\text { Mangeoire (sur } 2 \text { ans) } \\
\quad \text { Total des amortissements }\end{array}$ & $\begin{array}{r}10624 \\
8197 \\
3597 \\
715 \\
750 \\
13259\end{array}$ & $\begin{array}{l}0,9 \\
0,7 \\
0,3 \\
0,1 \\
0,1 \\
1,2\end{array}$ & $\begin{array}{r}10624 \\
8197 \\
3597 \\
715 \\
750 \\
13259\end{array}$ & $\begin{array}{l}0,8 \\
0,6 \\
0,3 \\
0,1 \\
0,1 \\
1,0\end{array}$ \\
\hline $\begin{array}{l}\text { 4. Recettes (chiffre d'affaires) } \\
\text { Courses ( } 312 \text { jours de travail)** }\end{array}$ & 1123200 & 100 & 1310400 & 100 \\
\hline 5. Marge brute annuelle & 687076 & 61,2 & 867076 & 66,2 \\
\hline 6. Marge nette annuelle & 673817 & 60,0 & 853817 & 65,2 \\
\hline 7. Recette journalière & 3600 & - & 4200 & - \\
\hline 8. Marge brute journalière & 2202 & 61 & 2779 & 66 \\
\hline 9. Marge nette journalière & 2160 & 60 & 2737 & 65 \\
\hline
\end{tabular}

* FCFA $=$ francs CFA

** 12 courses à 300 FCFA (fiacres) et 7 courses à 600 FCFA (charrettes)

Cas d'un propriétaire car le salaire du cocher n'est pas pris en compte

trop longue utilisation et au rythme élevé de leur exploitation. Compte tenu des tarifs pratiqués, les propriétaires rognaient sur l'entretien de leur animal et sur celui de l'attelage, ce qui expliquait le mauvais état des chevaux et le recours au matériel d'occasion. De plus, l'éclairage public urbain n'étant pas généralisé, le défaut de signalisation (catadioptres et éclairage) rendait les charrettes dangereuses à la tombée de la nuit.

\section{Résultats économiques}

Avec des chiffres d'affaires moyens quotidiens respectifs de 3600 et 4200 FCFA pour un travail de six jours par semaine, les fiacres et les charrettes ont généré une marge brute quotidienne moyenne de 2202 et 2779 FCFA, soit 61 et 66 p. 100 de leur chiffre d'affaires quotidien. Les charrettes sont apparues légèrement plus rentables car leurs tarifs étaient plus élevés. Bien qu'ayant la possibilité de faire plus de courses, les fiacres étaient astreints à des tarifs plus bas car ils étaient évidemment fortement concurrencés par les autres moyens de transport. Cependant, les tarifs des fiacres dont l'itinéraire dépendait de la destination de l'usager devenaient compétitifs par rapport aux tarifs du transport urbain dès que l'usager s'écartait des axes routiers fréquentés par les taxis clandos et les cars rapides. Les charrettes, quant à elles, sont restées sans concurrent réel pour le transport des fardeaux et des charges intermédiaires dans les quartiers.

La marge brute ou gain net monétaire pouvait ainsi atteindre 687000 FCFA par an pour un fiacre. Ce montant représentait 5,5 fois le capital investi (cheval, attelage et accessoires), en moyenne 124500 FCFA. Pour une charrette, la marge brute était de 867000 FCFA, soit sept fois le capital investi qui était dans ce cas évalué à 121150 FCFA. En prenant la valeur modale des versements aux propriétaires qui était de 1500 FCFA par jour, le capital correspondait à trois mois d'activités, si les versements étaient entièrement destinés à son recouvrement. Il s'ensuit un coût 
d'opportunité du capital nettement plus intéressant qu'avec l'épargne bancaire qui rapportait au mieux 8 à 9 p. 100 par an au moment de l'enquête. Depuis 1995, cette tendance s'est renforcée avec la baisse du taux d'épargne bancaire à 3,5 p. 100 et la stabilisation à 5,5 p. 100 de l'inflation qui avait été très forte $(30$ p. 100) après la dévaluation du franc CFA.

Si l'on prend deux chevaux par attelage, le gain quotidien pourrait être évalué entre 1200 et 1500 FCFA pour un même nombre de courses. En réalité, l'utilisation de deux chevaux était plus courante en période de forte demande comme pendant les fêtes. L'analyse a été limitée ici au cas de l'attelage avec un seul cheval.

La traction hippomobile était un secteur générateur d'emplois rémunérés à 36000 FCFA pour 24 jours de travail. Cette rémunération correspondait à une rémunération horaire de 187,5 FCFA, soit 10 p. 100 de moins que le salaire minimum interprofessionnel garanti (209,10 FCFA). Elle correspondait aussi à 2 p. 100 de plus que le salaire minimum agricole garanti (182,95 FCFA). De tels rapports expliquent l'attrait que représentait le travail de cocher pour les jeunes ruraux, surtout pendant les mois de saison sèche. A ce titre, la traction hippomobile a fourni près de 700 emplois à Thiès alors que le secteur concurrent des taxis urbains a concerné 500 chauffeurs de taxis. Les cars rapides, au nombre de 70, ont certainement occupé trois fois plus de personnes (chauffeurs et aides ou « apprentis »).

En plus d'un niveau appréciable de valorisation d'une main d'œuvre non qualifiée, le transport hippomobile urbain a entraîné des effets additionnels indirects sur les marchands d'aliments, les fabricants de charrettes et de harnais, sans oublier la satisfaction de la demande des usagers. Le niveau du gain net journalier estimé était relativement faible et il a entraîné un conflit d'intérêts inévitable étant donné les sommes de 1000 à 1500 FCFA par jour réclamées par les propriétaires. Un tel conflit a expliqué la forte tendance des cochers à satisfaire toute demande de transport nonobstant l'état du cheval. Par ailleurs, le poste alimentaire représentait 33 et 28 p. 100 du chiffre d'affaires respectivement pour les fiacres et les charrettes (tableau VI). Son niveau peut expliquer la tendance des propriétaires à rogner sur les dépenses en aliments et à ne pas satisfaire correctement les besoins d'entretien et de production des chevaux.

Il ressort de cette étude que la traction hippomobile est une prestation de service rentable quand sa demande est stable. Les hypothèses présentées dans le tableau VII permettent de donner des indices réalistes sur l'importance économique du transport hippo- mobile. Le transport hippomobile apparaît comme un secteur économique important s'il fonctionne pleinement et même s'il ne fonctionne qu'à 50 p. 100 de ses potentialités (156 jours de travail par an au lieu de 312). Il peut générer, en outre, de substantiels appoints pour les recettes municipales de l'ordre de 12 à 18 millions de FCFA (tableau VII). Ces résultats attestent du rôle économique de la traction hippomobile en plus de ses effets sociaux induits.

\section{Recommandations}

Il faut souligner que le transport hippomobile rencontre des difficultés qui limitent ses performances et sa participation à l'économie nationale. Il importe donc que des mesures idoines soient prises par les différents partenaires de la filière de la traction hippomobile urbaine. Les recommandations ci-dessous peuvent être faites par ordre de priorité aux principaux intéressés.

Propriétaires : hygiène des points de repos et écuries, amélioration de l'alimentation et son adaptation aux dépenses énergétiques, soins réguliers des chevaux, vaccinations et déparasitage, mise au travail à 4 ans, hygiène de travail et du harnachement, amélioration et entretien des attelages (le coût de ces mesures n'étant pas compensé par des recettes supplémentaires ni par une réduction des coûts de l'investissement, il y aura lieu de prévoir des mesures incitatrices), sensibilisation des cochers au code de la route, organisation en syndicat des cochers et des propriétaires pour la gestion de leurs activités.

Service vétérinaire et Bureau du cheval : relevé statistique régulier, amélioration des conditions d'accès au crédit, bonne vulgarisation des visites sanitaires et du livret signalétique et sanitaire, vaccinations obligatoires (peste équine, tétanos), prophylaxie de la lymphangite, conception et vulgarisation d'un système d'assurance.

Autorités communales et police : améliorations juridiques (réglementation, taxes), abandon du harcèlement et de la répression, utilisation d'une partie des taxes collectées pour des actions spécifiques en faveur de la traction hippomobile (santé, amélioration génétique).

Recherche : indicateurs de conformation ou note d'état corporel adaptés pour la traction hippomobile et le travail agricole, programme d'amélioration génétique (caractères pour la traction), sérosurveillance, adaptation des attelages (charrettes et fiacres), messages adaptés pour l'alimentation (formulation de rations).

\section{Tableau VII}

Importance économique de la traction hippomobile à Thiès (x 1000 FCFA)

\begin{tabular}{|c|c|c|c|c|c|c|c|c|}
\hline \multirow{2}{*}{$\begin{array}{l}\text { H ypothèses } \\
\text { Attelages }\end{array}$} & \multicolumn{4}{|c|}{312 jours de travail par an } & \multicolumn{4}{|c|}{156 jours de travail par an } \\
\hline & CA & MB & Taxes $^{1}$ & Taxes $^{2}$ & $\mathrm{CA}^{3}$ & MB 4 & Taxes $^{1}$ & Taxes $^{2}$ \\
\hline $\begin{array}{l}\text { Fiacres } \\
n=450\end{array}$ & 505440 & 309184 & 10800 & 6480 & 252720 & 564642 & 10800 & 6480 \\
\hline $\begin{array}{l}\text { Charrettes } \\
n=250\end{array}$ & 327600 & 216769 & 7800 & 4680 & 163800 & 529690 & 7800 & 4680 \\
\hline $\begin{array}{l}\text { Total } \\
n=700\end{array}$ & 833040 & 525953 & 18600 & 11160 & 416520 & 109433 & 18600 & 11160 \\
\hline
\end{tabular}

1. Hypothèse : taxes à 100 p. 100 de recouvrement

2. Hypothèse : taxes à 50 p. 100 de recouvrement

3. $\mathrm{CA}=$ chiffre d'affaires

4. $\mathrm{MB}=$ marge brute 


\section{CONCLUSION}

L'analyse de l'état du transport hippomobile face à l'hégémonie de ses coûteux substituts modernes et la compréhension de ses perspectives économiques au Sénégal peuvent contribuer à mieux éclairer les options qui sont élaborées pour réajuster la structure des économies africaines et promouvoir leur réel développement.

Pour un travail de six jours par semaine, les fiacres et les charrettes génèrent un gain monétaire net quotidien moyen de 2202 et 2779 FCFA. Une telle marge brute permet aux propriétaires et aux cochers d'avoir une activité rémunératrice dans un contexte fortement affecté par le chômage. La rémunération équivaut à seulement 10 p. 100 de moins que le salaire minimum interprofessionnel garanti et aussi à 2 p. 100 de plus que le salaire minimum agricole garanti.

Considérée comme un mal nécessaire, une nuisance et une source de dérangement voire d'insécurité sur les axes routiers urbains, la traction hippomobile reste toujours un service utile pour une clientèle urbaine provenant de toutes les couches sociales. Grâce à son accessibilité, à la modicité des tarifs pratiqués et enfin à la disponibilité des cochers à sillonner tous les quartiers, les fiacres et les charrettes resteront longtemps encore une composante de la circulation urbaine, surtout dans les villes secondaires du Sénégal. Une plus grande tolérance et un soutien approprié des pouvoirs publics et des autorités communales permettraient d'exploiter plus efficacement leurs potentialités et leur importance économique.

\section{BIBLIO GRAPH IE}

1. CAMARA B., 1994. Economie du transport hippomobile urbain. Cas de la commune de Rufisque. Mémoire, Ecole nationale des cadres ruraux de Bambey, Bambey, Sénégal, $69 \mathrm{p}$.

2. DE FRANCO H.C., 1905. Etude sur le cheval en Afrique occidentale. Melun, France, Imprimerie administrative, $109 \mathrm{p}$.

3. DIRECTION DE L'ELEVAGE, 1993. Plan d'action de l'élevage équin. Dakar, Sénégal, Ministère de l'agriculture, 26 p.

\section{Summary}

Ly C., Fall B., Camara B., N diaye C.M. U rban draft horses in Senegal. Situation and economic value in the city of Thies

The study describes the situation and economic value of draft horses in urban and suburban Senegal, with a focus on the city of Thies. O ne hundred horse cars (64 cabs and 36 carts) were randomly chosen and their drivers interviewed. The data were about drivers, horses, characteristics and management of carts and also economic results (costs and returns). For six working days a week, cabs and carts generated for their owners an average daily monetary net gain of about $61 \%$ (2202 CFA francs) and 66\% (2779 CFAF) of their daily turnover (3600 and 4200 CFAF, respectively). Carts seemed slightly more profitable since their fares were higher. The economic worth of the horses is shown and recommendations are made to improve performances and to integrate them into the national economy.

Key words: Horse - Transport - Urban area - Economic value Animal drawn vehicle - Cart - Hitch - Cost benefit analysis Senegal.
4. DIIMADOUM J., 1994. Dominantes pathologiques chez les chevaux de trait urbains dans la région de Dakar. Résultats d'une enquête clinique. Thèse méd. vét., EISMV, Dakar, Sénégal, 63 p. ( $\left.n^{\circ} 19\right)$

5. DOUTRESSO ULLE G., 1947. L'élevage en Afrique occidentale française. Paris, France, Editions Larose, 298 p.

6. DOUTRESSO ULLE G., 1952. L'élevage au Soudan français. Son économie. Alger, Algérie, Imbert, $374 \mathrm{p}$.

7. EPSTEIN H., 1971. The origin of the domestic animals of Africa, vol. II. N ew York, USA, Africana Publishing, $719 \mathrm{p}$.

8. FALL A.B., 1988. Les Iymphangites équines au Sénégal Epidémiologie et étiologie. Thèse méd. vét., EISMV, Dakar, Sénégal, 93 p. $\left(n^{\circ} 51\right)$

9. FAO, 1992. Annuaire de la santé animale. Rome, Italie, FAO.

10. FAYE A., 1989. Le rôle du cheval dans le développement rural en zone sahélo-soudanienne du Sénégal. L'exemple du sud du bassin arachidier. Cah. Rech. Dév., $21: 19-29$.

11. FIELDING D., 1991. The number and distribution of equines in the world. In: Fielding D., Pearson R.A. eds., D onkeys, mules and horses in tropical agricultural developement. Edinburgh, UK, University of Edinburgh, p. 62-66.

12. JARRIGE R., MARTIN-ROSSET W., eds., 1984. Le cheval. Reproduction, sélection, alimentation, exploitation. Paris, France, Inra, $350 \mathrm{p}$.

13. LARRAT R., 1947. L'élevage du cheval au Sénégal. Revue Elev. Méd. vét. Pays trop., 1 : 257-265.

14. LAVALARD E., 1894. Le cheval dans ses rapports avec l'économie rurale et les industries de transport. Paris, France, Librairie de FirminDidot, $938 \mathrm{p}$.

15. NDIAYE M., 1978. Contribution à l'étude de l'élevage du cheval au Sénégal. Thèse méd. vét., EISMV, Dakar, Sénégal, 183 p. ( $\left.n^{\circ} 15\right)$

16. NDOYE D.P., 1988. Le cheval de course au Sénégal. Thèse méd. vét., EISM V, Dakar, Sénégal, 126 p. ( $\left.n^{\circ} 29\right)$

17. PECAUD J., 1927. Les chevaux de notre colonie du Tchad. Paris, France, L. Fournier, $55 \mathrm{p}$.

18. SPSS, 1994. Statistical package for the social sciences. Chicago, IL, USA, SPSS.

Accepté le 2.10 .98

\section{Resumen}

Ly C., Fall B., Camara B., Ndiaye C.M. El transporte urbano con equinos en Senegal - Situación e importancia económica en la ciudad de Thies

El presente estudio describe la situación y analiza la importancia económica de la tracción urbana y peri urbana generada por equinos en Senegal, a partir del caso de la ciudad de Thies. Se escogieron al azar cien atelajes (64 coches y 36 carretas) y se interrogó a los cocheros. Los datos recolectados concernieron a los cocheros y a los caballos, las características y el manejo de los atelajes y, finalmente, los resultados económicos (costos, recetas y ganancias). Para un trabajo de seis días por semana, los coches y las carretas generaron, para el propietario del atelaje, una ganancia monetaria neta cotidiana media equivalente a 61\% (2 202 francos CFA) y $66 \%$ (2 779 FCFA) del respectivo monto de negocio diario (3 600 y 4200 FCFA). Las carretas parecen ser ligeramente más rentables, ya que las tarifas fueron mas elevadas. La importancia económica del caballo se ilustra y se dan recomendaciones para mejorar los rendimientos y la participación en la economía nacional.

Palabras clave: Caballo - Transporte - Zona urbana - Valor económico - Vehículo de tracción animal - Carreta Enganche - Análisis de costos y beneficios - Senegal. 